\title{
Modeling of a Wave Farm Export Cable for Electro-Thermal Sizing Studies
}

\author{
Charles-Henri Bonnard $^{\mathrm{a}, *}$, Anne Blavette ${ }^{\mathrm{a}}$, Salvy Bourguet ${ }^{\mathrm{b}}$, Adrien \\ Charmetant ${ }^{\mathrm{c}}$ \\ ${ }^{a}$ SATIE, CNRS, Université de Rennes, ENS-Rennes, 35170 Bruz, France \\ ${ }^{b}$ IREENA, Université de Nantes, 37 Boulevard de l'Université, 44602 Saint-Nazaire, France \\ ${ }^{c}$ Nexans RछD, Nexans Research Center, 29 rue Pré Gaudry, 69007 Lyon, France
}

\begin{abstract}
So far, only few studies have addressed the techno-economic optimization of an export cable sizing in the specific case of wave energy farms. However, in these works, the cable current rating is determined based on conservative steady-state conditions regarding the farm current output whereas considering dynamic conditions may be more relevant in the case of wave energy applications. However, this implies developing and using dedicated electro-thermal models, which poses a challenge regarding the determination of the modeling fineness level to be adopted for such studies. Hence, this paper presents several numerical models, the most refined of which is compared with experimental data, as well as well as preliminary cable sizing studies. Contrary to previous works in this field, the fluctuating nature of wave energy is considered here, thus allowing for more realistic results.
\end{abstract}

Keywords: Submarine export cable; electro-thermal model; wave energy farm

\section{Introduction}

Over the past decades, wave energy has been shown to have a great potential in terms of electricity generation. Hence, it constitutes a relevant alternative to non-renewable sources of energy. However, the levelized cost of energy (LCOE)

\footnotetext{
* Corresponding author

Email address: charles-henri.bonnard@ens-rennes.fr (Charles-Henri Bonnard)
} 
5 is greater for wave energy than for more conventional power sources [1], [2]. Consequently, significant cost reductions are required for wave energy to become economically attractive and to allow its grid integration on a large-scale. Among the potential options available to reduce the wave energy LCOE, optimizing the electrical infrastructure of wave farms, and especially their cabling infrastructure, was identified as a relevant target in order to reduce capital expenditure. Based on the experience of the offshore wind energy industry [3, 4, 5], it is indeed interesting to note that the costs related to the electrical infrastructure, and composed mostly of cabling and substations costs (approx. 50\% each), may represent up to $20 \%$ of a wave farm capital expenditure. Hence, a reasonable percentage of cost reduction in this area may lead to a significant decrease in terms of capital expenditure [6, 7].

However, so far, and to the best of the authors' knowledge, only few studies have addressed the techno-economic optimization of the cabling infrastructure sizing in the specific case of wave energy farms [6, 7]. In both studies, the cable current rating is determined based on an approximation implying that a wave farm outputs its maximum current in the form of a constant profile, thus implying steady-state conditions. However, this may lead to a highly conservative estimation of a cable current rating in the specific case of wave energy. This 25 power source is indeed highly fluctuating by nature and its variations are reflected in the power output of a wave farm. This can be explained by the fact that most wave energy converters (WECs) are oscillating bodies with limited storage capacities, thus generating a fluctuating current output. Consequently, a farm composed of such devices is also expected to output a fluctuating current profile, which is however potentially reduced thanks to the devices aggregation effect. Hence, determining the current rating of a cable based on more realistic, dynamic conditions could lead to a dramatic decrease regarding its sizing compared to the case where conservative, steady-state conditions are considered, as mentioned in preliminary works by the authors [8, 9].

In order to perform such techno-economic optimization studies focusing on 
the thermal response of a cable to a fluctuating current profile representative of wave energy conditions, dedicated electro-thermal models are required. However, developing such models presents challenges regarding the determination of the modeling fineness level to be adopted for the type of applications considered 40 in this paper. There exist indeed several approaches to determine the thermal response of a cable, each of which presents different performance levels in terms of computational burden and of error level. The less computationally intensive approach is based on relatively simple models described in standards, such as IEC standard 60287-1-1 [10]. In this document, the current rating is determined based on steady-state conditions where the amplitude of the current input to the cable is considered as constant over time. However, as explained earlier, using the maximum current output by the farm in such an approach is quite conservative in the case of wave energy applications. Although a complementary standard was developed to cover the case of a cycling load [11, it may not be relevant here. This standard includes indeed several approximations which do not allow to study a cable thermal response to a current profile fluctuating at the timescale of seconds.

Alternatively, the finite element method (FEM) is a far more computationallyintensive approach, but from which accurate results can be obtained with a userdefined level of approximation. For instance, a cable may be modeled in two or three dimensions (2D or 3D), and may include either linear or non-linear thermal characteristics for resistivity, specific heat and thermal conductivity. In this paper, the results obtained from different models, presenting different levels of modeling fineness (standard-based/FEM-based, 2D/3D and using linear/nonlinear thermal characteristics in the case of FEM-based models) will be presented. This study is intended to provide preliminary results regarding the level of error as a function of the level of modeling fineness. It is also important to mention that experiments, whose protocol will be described later in the paper, were carried out in order to validate the numerical results.

Beside providing preliminary guidance regarding the modeling fineness, this paper is also intended to present results on preliminary cable sizing studies 
focusing on two aspects. The first one regards the critical design conditions (either normal or short-circuit ones) for cable design. Both these studies are indeed usually performed at the planning stage for more conventional types of applications to determine which conditions constitute the critical design ones, therefore imposing a lower bound on the cable sizing. The second investigated aspect regards the potential room for improvement concerning the cable sizing when more realistic, dynamic conditions are considered, as opposed to conservative steady-state conditions.

Hence, in order to combine these two objectives, i.e. assess the error level as a function of the modeling fineness level, and carry out preliminary studies on cable sizing, the developed numerical models are used in two case studies which are presented in this paper. The obtained results are used to draw conclusions for each of the two topics considered here.

The rest of the paper is organized as follows: Section 2 presents the different numerical models developed as part of this study. Then, Section 3 details the experiments carried out on a real submarine cable to be used as a benchmark for the results obtained through numerical simulations. Following this, Section 4 describes the two case studies (considering either normal or short-circuit conditions) while Section 5 details the results. Finally, Section 6 concludes the paper.

\section{Numerical models}

This section presents in the first subsection the characteristics of the cable considered here. Then, it describes the developed numerical models, which are based either on IEC standards or on the finite element method (FEM).

\subsection{Power cable characteristics}

In this paper, numerical and experimental studies were carried out based on a real, 5.5 meter-long submarine cable sample. This sample was provided as a courtesy of École Centrale de Nantes (ECN). It corresponds to an unused part of 


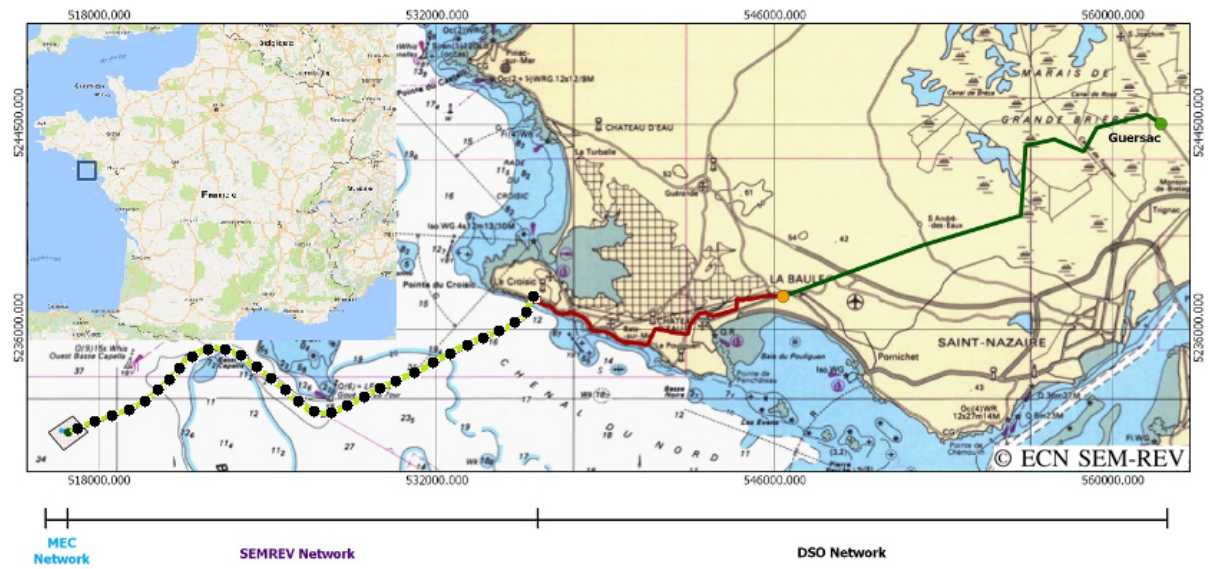

Figure 1: Map indicating the localization of SEM-REV test site. The export cable connects the SEM-REV offshore hub to an onshore substation and to the local network belonging to French DSO Enedis (red and dark green paths). Figure modified, courtesy of École Centrale de Nantes 12 .

the cable which ECN acquired for its SEM-REV offshore test site located off Le Croisic, France [12. Twenty-four kilometers of this cable are currently installed at the SEM-REV test site and used as an export cable buried 1.5 meter deep in the soil. This export cable follows the green, dotted path shown in Fig. 1. The cable connects an onshore electrical substation (managed by French distribution system operator (DSO) "ENEDIS") to the hub of the SEM-REV test site where three devices (e.g. wave energy converters (WECs) and wind turbines) can be connected simultaneously. A cross-sectional view of a small sample of this 3-phase, 20-kV, 8-MVA cable, whose architecture is typical of static cables, is shown in Fig. 2. It is composed of $3 \times 95 \mathrm{~mm}^{2}$ copper conductors. For each phase, the conductor is surrounded by a semiconducting polymer screen, a cross-linked polyethylene (XLPE) insulation, a second semiconducting screen, a copper screen and finally an external sheath made of polyethylene (PE). The three phases are arranged in a twisted trefoil shape. The space between each phase and the inner sheath, also called "filler", is made of a combination of bulk PE tubes, PE yarn and air. Optical fibers are also present. The armour constitutes the last layer and is made of galvanized steel wires sheathed with 


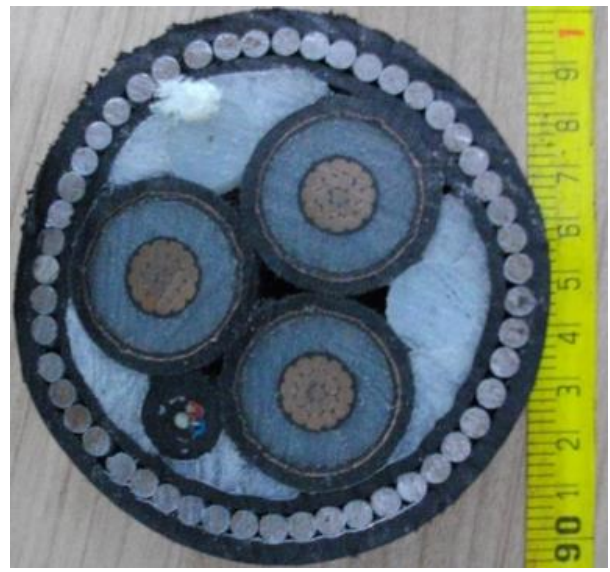

Figure 2: Cross-sectional view of a small cable sample. Its architecture is typical of submarine export cables.

PE. According to the electric insulation used in this cable, the maximum operating temperature of the copper cores is equal to $90^{\circ} \mathrm{C}$. However, it is allowed to reach $250{ }^{\circ} \mathrm{C}$ in case of a 5 s-long short-circuit, which is the longest short-circuit duration to be considered for cable design purposes, as recommended in IEC standard 60949 [13. Finally, the cable current rating $I_{R}$, based on steady-state conditions, is equal to $290 \mathrm{~A}$. The following sections will present the numerical models (IEC standard-based and FEM-based) of the cable presented in the current section.

\subsection{IEC standard-based model}

IEC standard 60287-1-1 provides the following formula for calculating the temperature rise $\Delta T$ above ambient temperature of a three-phase buried cable:

$$
\begin{aligned}
\Delta T= & \left(I^{2} R+0.5 W_{d}\right) R_{T 1}+\left[I^{2} R\left(1+\lambda_{1}\right)+W_{d}\right] n R_{T 2} \\
& +\left[I^{2} R\left(1+\lambda_{1}+\lambda_{2}\right)+W_{d}\right] n\left(R_{T 3}+R_{T 4}\right)
\end{aligned}
$$

where $I$ is the current flowing in one conductor, $R$ is the alternating current resistance per unit length of the conductor operating temperature, $W_{d}$ is the dielectric loss per unit length for the insulation surrounding the conductor, $R_{T 1}$ 
is the thermal resistance per unit length of between one conductor and the sheath, $R_{T 2}$ is the thermal resistance per unit length of the bedding between sheath and armour, $R_{T 3}$ is the thermal resistance per unit length of the external serving of the cable, $R_{T 4}$ is the thermal resistance per unit length between the cable surface and the surrounding medium, $n$ is the number of load-carrying conductors in the cable, $\lambda_{1}$ is the ratio of losses in the metal sheath to total losses in all conductors, and $\lambda_{2}$ is the ratio of losses in the armouring to total losses in all conductors in the cable. Complementary formulae are provided in IEC standards $60287-1-1$ and $60287-2-1$ [14 to calculate parameters $R$ to $\lambda_{2}$, and were used for the numerical simulations presented in this paper. The thermal characteristics of the cable-soil system (resistivity, specific heat, and thermal conductivity) were modeled linearly, as proposed in the two standards. Contrary to the previous works mentioned earlier, where current $I$ was set to the maximum current output of a wave farm, current $I$ is here set to the rootmean-square (RMS) over the simulation period of the RMS AC current profile. For the sake of illustration, an RMS AC current profile is shown in Fig. 6 it is the RMS value of such a profile to which current $I$ is set here. Considering the RMS value of the RMS current profile is envisaged to be more relevant in terms of temperature estimation than focusing on the maximum current. Hence, the relevance of such an approach will be investigated in the paper.

\subsection{Finite Element Method models}

Contrary to the previous approach based on IEC standards, simulating a model using the Finite Element Method (FEM) can be quite demanding in terms of computing resources, especially for 3D models combining several coupled physics. Hence, such 3D models are usually reduced to 2D models whenever possible. This strategy has been applied here, and two 2D models were developed: one includes linear thermal charateristics for the resistivity, specific heat and conductivity, as in our previous study [9], while the other includes more refined, non-linear characteristics. The non-linear characteristics for the second model were found in [14] 15]. These two models are presented in this section 
and a comparative analysis with the experimental results, intended for validation purposes, is described in Section 5.2. It is important to note that the filler is a mix of air and PE yarn with a usually unknown mixing ratio, thus making more difficult the prediction of its thermal behavior. Hence, simulations were performed considering different values for the mixing ratio, from a filler solely made of PE to a filler composed exclusively of air.

The 2D cable models were developed using the well-known COMSOL Multiphysics $\mathbb{R}^{2}$ simulation software [16]. The thermal problem was implemented using the general heat equation, i.e.

$$
\rho_{m} C_{p}(T) \frac{\partial T}{\partial t}=\nabla \cdot(\kappa(T) \nabla T)+Q_{J},
$$

where $\rho_{m}$ is the mass density of each material, $C_{P}$ is the specific heat, $T$ is the temperature, $t$ is the time, $\kappa$ is the thermal conductivity and $Q_{J}=\int_{V} \rho_{e} J^{2} d V$ is the total Joule losses inside the cable computed from the electromagnetic part of the model. Term $J$ is the electric current density. The convection in free air was modeled by means of a non-linear law of cooling, and the cooling power $Q_{c}$ is defined as:

$$
Q_{c}=h\left(T_{s}-T_{r}\right) \cdot S \cdot\left(T_{s}-T_{r}\right)
$$

where $T_{s}$ is the temperature of the cable external sheath surface, $T_{r}$ is the room temperature, $S$ is the external sheath area, and $h\left(T_{s}-T_{r}\right)$ is an effective non-linear convection coefficient based on formulae given in [17] and taking into account a long horizontal circular cylinder. The radiative heat transfer $Q_{r}$ has also been considered and follows the Stefan-Boltzmann law, i.e.:

$$
Q_{r}=\epsilon \cdot \sigma_{T} \cdot S \cdot\left(T_{s}^{4}-T_{r}^{4}\right)
$$

where the emissivity factor $\epsilon$ was selected as equal to 0.9 in this study, and $\sigma_{T}$ is the Stefan-Boltzmann constant. The electromagnetic problem has been implemented using Maxwell's equations, i.e.

$$
\nabla \times H=J,
$$




$$
\begin{gathered}
B=\nabla \times A, \\
E=-\frac{\partial A}{\partial t}, \\
J=\sigma_{e} E,
\end{gathered}
$$

where $H$ is the magnetic field, $B$ is the magnetic flux density, $A$ is the vector potential, $E$ is the electric field and $\sigma_{e}$ is the electrical conductivity.

\section{Experimental setup and protocol}

This section presents the experimental setup used as a benchmark for the numerical simulations carried out in this study. Then, the experimental protocol is described.

\subsection{Experimental setup}

As mentioned earlier, experiments were performed using a 5.5 meters-long sample of the SEM-REV export cable. In order to avoid any thermal effect from the ground during our experiments, the cable was installed in free air $1 \mathrm{~m}$ above the floor as presented in Fig. 3. A voltage-controlled current source was used to power the cable. It is designed to reach a maximum current of $900 \mathrm{~A}$ in steady-state (AC or DC) in a single-phase configuration [18 [19]. Using it as a three-phase power source limits the maximum current value to $3 \times 300 \mathrm{~A}$.

\subsection{Experimental protocol}

The characteristics of the three cases considered for the experiments are shown in Table 1. In Cases 1 and 3, the amplitude of the current profile is constant. They were introduced to provide benchmark values for the numerical computation of the losses in $\mathrm{DC}$ and $\mathrm{AC}$, as well as for the correct evaluation of the heat transfer from the conductor to the surrounding air. On the contrary,

in Case 2, two variable, pulsed profiles, shown in Fig. 4. are used. The first one is used to power a single phase while in the second scenario of Case 2, all 


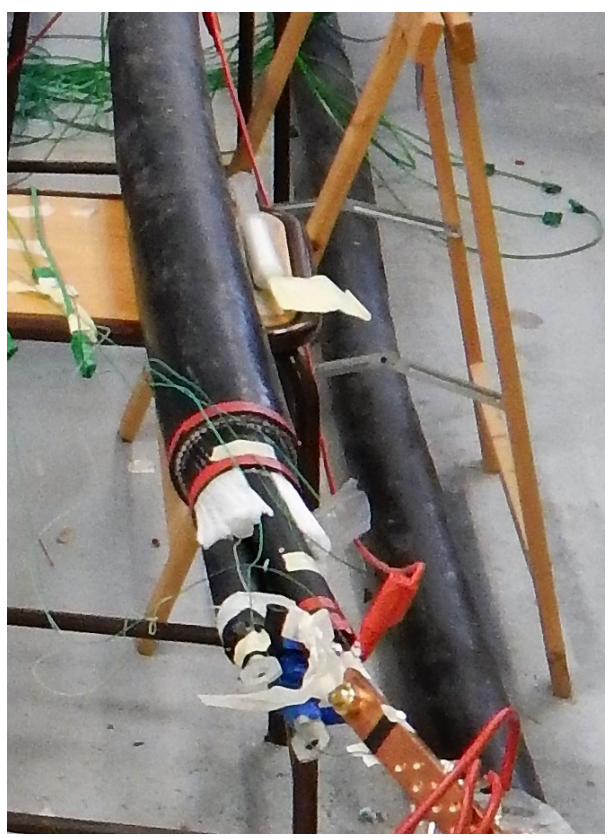

Figure 3: Photograph of the instrumented cable installed in free air

Table 1: Characteristics of the three cases considered for the experiments

\begin{tabular}{|c|c|c|c|}
\hline Case & Current profile & Amplitude & $\begin{array}{c}\text { Number of } \\
\text { powered phases }\end{array}$ \\
\hline 1 & Steady-state DC current & $295 \mathrm{~A}$ & 1 \\
\hline 2 & Pulsed DC current & $150-600 \mathrm{~A}$ & 1,3 \\
\hline 3 & Steady-state AC current $(50 \mathrm{~Hz})$ & $200 \mathrm{~A}_{R M S}$ & 3 \\
\hline
\end{tabular}




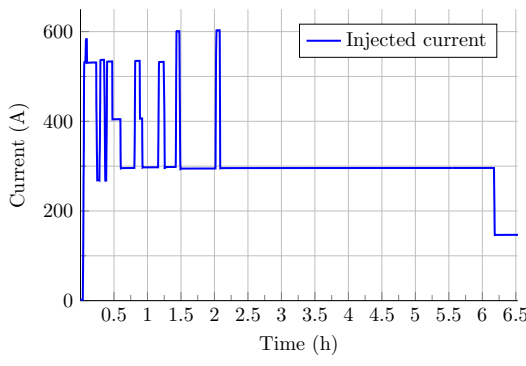

(a) Powering 1 phase

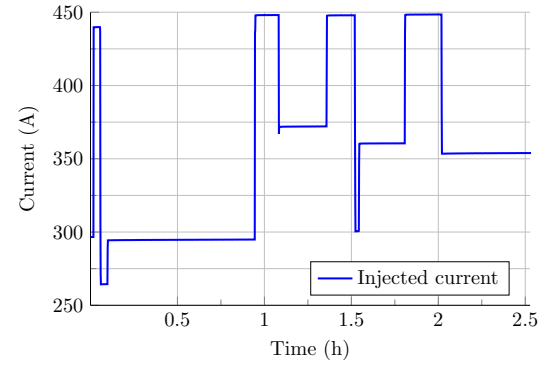

(b) Powering 3 phases

Figure 4: Pulsed DC current profiles applied in Case 2.

temperature of the powered phase(s) over time, using the temperature dependance of the copper core resistivity $\rho_{e}$, which can be expressed as [10]:

$$
\rho_{e}(T)=\rho_{0} \times\left[1+\alpha\left(T_{c}-T_{0}\right)\right],
$$

where $\rho_{0}=1.724 \times 10^{-8} \Omega . \mathrm{m}, \alpha=3.93 \times 10^{-3} \mathrm{~K}^{-1}$ is the temperature

coefficient of copper, $T_{c}$ is the core temperature in $\mathrm{K}$ and $T_{0}=293.15 \mathrm{~K}$ is the reference temperature for which $\alpha$ is specified. The time-resistance $R$ of the powered phase was evaluated using a classical four-point probes method with voltage and current measurements. The core cross-section, the twisting pitches of both strands and phases, directly impact the real length of the copper. Thus, using $R$ and considering all the physical parameters that influence the core resistance, we were able to determine its resistivity $\rho_{e}$. Once the value of $\rho_{e}$ is calculated, we were able to get back to the conductor core temperature by using Equation 9. It is also worth mentioning that the cable was powered during several hours prior to the experiments so that it had reached steadystate conditions when the monitoring phase started.

\section{Case studies description}

This section details the two case studies performed in order to evaluate which type of conditions (normal or short-circuit) constitutes the critical design 


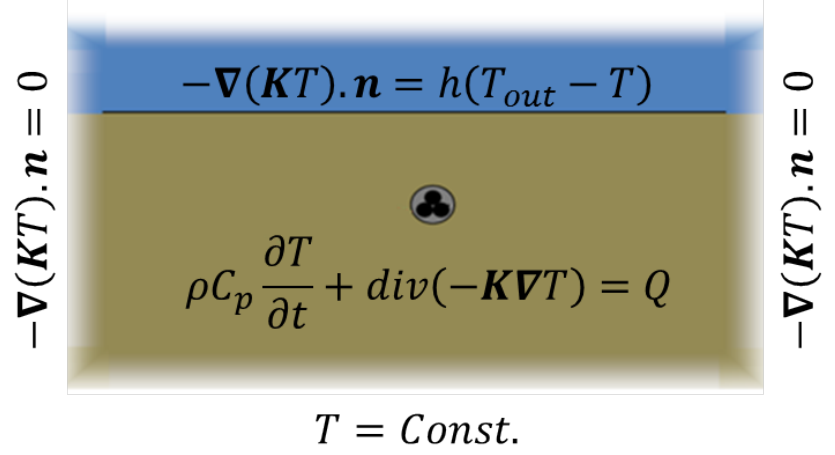

Figure 5: Illustration of the boundary conditions

conditions regarding cable sizing, from an electro-thermal perspective, and in order to assess the potential room for improvement regarding the cable sizing when dynamic (normal) conditions are considered. Both case studies are based on numerical simulations using the most refined model presented in the study, i.e. the 2D FEM model including non-linear thermal characteristics (sometimes referred to as "non-linear 2D FEM model" in the rest of the paper for the sake 235 of brevity).

In both case studies, and similarly to the physical installation at the SEMREV test site, the cable is considered as buried $1.5 \mathrm{~m}$ deep under the sea bed. The boundary conditions depicted in Fig. 5 were used in the thermal problem. The ambient temperature for both the sea and the soil was selected as equal to $12{ }^{\circ} \mathrm{C}$. This is typical of sea temperature conditions in wave energy abundant northwestern Europe [20].

\subsection{Case study 1: normal conditions}

A sea-state with a relatively high energy level is considered, as it presents a significant wave height $H_{s}$ equal to $6 \mathrm{~m}$ and a peak period $T_{p}$ equal to $9 \mathrm{~s}$. A wave farm composed of 15, 20 or 25 WECs (three configurations are covered) is considered here. Its devices are identical heaving buoys controlled passively, as detailed in [21. The current profile injected into the cable, when the 20 WECs 


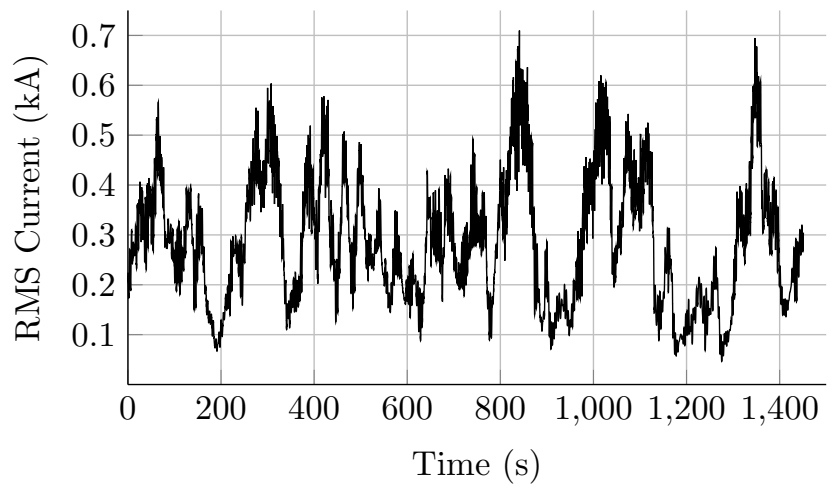

Figure 6: Elementary sequence of the periodic current profile amplitude (RMS) for a 20 WECs farm used for Case study 1

farm is considered, is shown in Fig. 6. It was computed based on a simulated wave energy farm described in a previous paper 22. The same methodology was applied to simulate the current output profiles of the 15 WECs, and of the 25 WECs configurations. As regards the 20 WECs farm case, retained for illustration purposes, it is interesting to note that the corresponding current profile shown in Fig. 6 fluctuates between $70 \mathrm{~A}_{R M S}$ and $700 \mathrm{~A}_{R M S}$ approximately, i.e. between $25 \%$ and $240 \%$ of the cable rated RMS current $I_{R}$. Such values can temporarily lead to a significant losses increase, i.e. nearly 6 times the normal operating losses, thus generating a significantly high amount of heat which may lead to the cable overheating. The current profiles were repeated sequentially to reach a simulation time of about 28 hours, i.e. the duration necessary to reach equilibrium conditions for the thermal problem. Note that the RMS values of the RMS current computed over the entire profile duration (i.e. $1426 \mathrm{~s}$ ) are equal to $245 \mathrm{~A}_{R M S}$ (15 WECs farm case), $315 \mathrm{~A}_{R M S}$ (20 WECs farm case), and $375 \mathrm{~A}_{R M S}$ (25 WECs farm case). The corresponding cable cores temperatures were simulated by means of the three different numerical models presented in Section 2 , 


\subsection{Case study 2: short-circuit conditions}

Short-circuit conditions must be taken into account in cable design, and in particular regarding its sizing. Under such conditions, the core temperature increases rapidly during a short period of time. However, it must remain below a maximum allowed limit, e.g. $250{ }^{\circ} \mathrm{C}$ for XLPE electrical insulation [23] and and PVC 24]. In this case study, short-circuits were considered to occur once an equilibrium state, corresponding to this of the 20 WECs scenario in Case study 1 , has been reached.

Note that the electrical grid architecture of a WEC farm may be expected to be very similar to this of offshore windfarms equipped with Type 4 wind turbines using fully rated back-to-back AC/DC/AC converters 25. Such electronic interface allows to limit the fault current to a value that is barely above the rated current 26]. Based on this observation, the contribution of the WEC farm to the short-circuit current can be neglected. It is therefore assumed that the magniand to a strong grid 27 . The fault current $I_{S C}$ was calculated considering the steady-state fault current and the DC offset current, i.e.

$$
I_{S C}=\frac{E_{U} \sqrt{2}}{Z_{U}}\left[\sin (\omega t-\phi)+\sin \phi \cdot e^{-\frac{R_{U}}{L_{U}} t}\right],
$$

where $E_{U}$ is the phase-to-phase voltage of the electrical network, $\phi$ is the phase shift between voltage and current such that $\phi=\tan ^{-1}\left(\frac{X_{U}}{R_{U}}\right)$. An $X / R$ ratio equal to 3 is representative of a weak grid whereas a ratio equal to 20 can be considered as representative of a strong grid [27, 28]. Although 3-phase short-circuits are usually the most critical cases, it is also important to consider 
single-phase short-circuits, as their consequences may, under certain conditions, be worse than for the former. For instance, an internal defect in the cable may lead the current to flow from the conductor directly to its copper screen which is grounded on its extremity. However, the cross-section of the core is greater than this of its copper screen (they are equal to $95 \mathrm{~mm}^{2}$ and $16 \mathrm{~mm}^{2}$ respectively for the cable considered in this study). Then, it is expected that the copper screen temperature increases faster than the core temperature for a given fault current. However, the copper screen temperature must still remain below the maximum allowed limit to prevent any excessive, global damage of the dielectric insulation. Thus, in this paper, two short-circuit scenarios were considered: a three-phase short-circuit scenario and a single-phase short-circuit scenario where a conductor is connected directly to its copper screen, as described previously.

\section{Results}

This section describes the results obtained for the two case studies performed for normal and short-circuit conditions. Then, it describes the results related to the modeling fineness analysis.

\subsection{Case studies results}

Case study 1 (i.e. considering normal conditions) was performed using the three different numerical models described in Section 2 while Case study 2 (i.e. considering short-circuit conditions) was performed using only the 2D FEM model including non-linear thermal characteristics. This model, as it will be detailed in Section 5.2.1, presents results similar to the experimental results and is therefore assumed as a relevant benchmark for the other two numerical models (i.e. IEC standard-based, and 2D FEM model including linear thermal characteristics) for the comparative analysis on modeling fineness described in Section 5.2 .2 . 


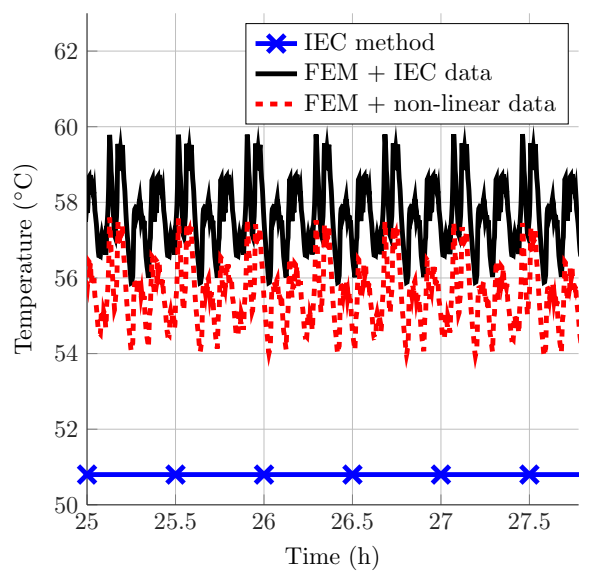

(a) 15 WECs.

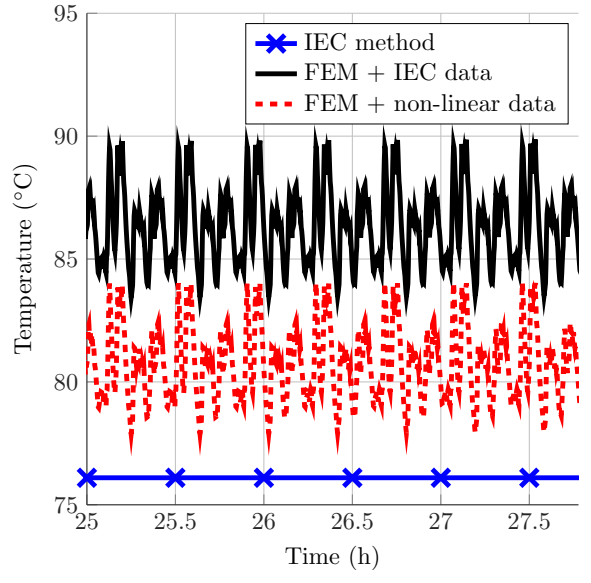

(b) 20 WECs.

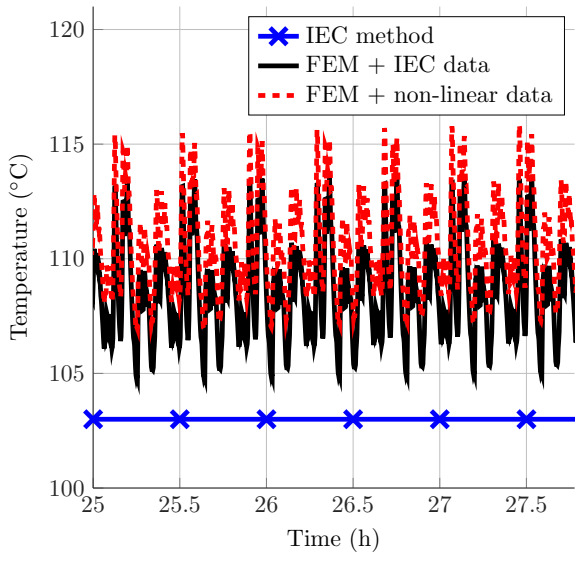

(c) 25 WECs.

Figure 7: Core temperatures simulated with the three numerical different models and for three different numbers of WECs

\subsubsection{Case study 1: normal conditions}

The results corresponding to the core temperature simulations can be seen in Fig. 7. In this section, only the results corresponding the non-linear 2D FEM model (referred to as "FEM + non-linear data" in the figure) will be discussed, and compared to the rated current $I_{R}$. The results corresponding 
to the other two models will analyzed as part of the comparative analysis on modeling fineness in Section 5.2 .2

It can be observed that, despite the high current peaks generated by the WEC farm (e.g. up to 2.5 times the rated current $I_{R}$ when the 20 WECs farm is considered), the thermal inertia of the whole system composed by the cable and the soil acts as a strong, natural damper which reduces the amplitude of the cable core temperature fluctuations to $7{ }^{\circ} \mathrm{C}$ only. Besides, still in the 20 WECs farm case, it can be observed that the thermal constraints are still satisfied as the core temperature does not exceed the maximum operating temperature of $90{ }^{\circ} \mathrm{C}$. This confirms that significant current peaks may be injected in a submarine export cable without impacting the cable nor its environment, at least from a thermal perspective. However, it is also important that the surrounding shortcircuit protection system (e.g. circuit breakers) is also properly designed to avoid any undesired shedding.

The results also show that the operating maximum temperature of $90^{\circ} \mathrm{C}$ is reached for the 20 WECs farm, whose RMS current is equal to $315 \mathrm{~A}_{R M S}$, as mentioned in Section 4.1. This means that a current profile whose RMS value is $9 \%$ greater than the rated current $I_{R}$ can be safely injected into the cable while still satisfying the thermal constraints. This figure needs of course to be refined through an extensive analysis, including different sea-states for instance. However, this study confirms that a wave farm export cable may be sized to

345 a dramatically lower value than the maximum current output by the farm (as considered in other works), thus leading to potentially significant savings.

\subsubsection{Case study 2: short-circuit conditions}

As mentioned earlier, Case study 2 is composed of two studies: one deals with a three-phase short-circuit while the other focuses on a single-phase shortcircuit. Both studies were performed using the non-linear 2D FEM model.

Three-phase short-circuit. According to IEC standard 60949 [13], a fault duration of up to 5 seconds should be considered for cable design purposes. 


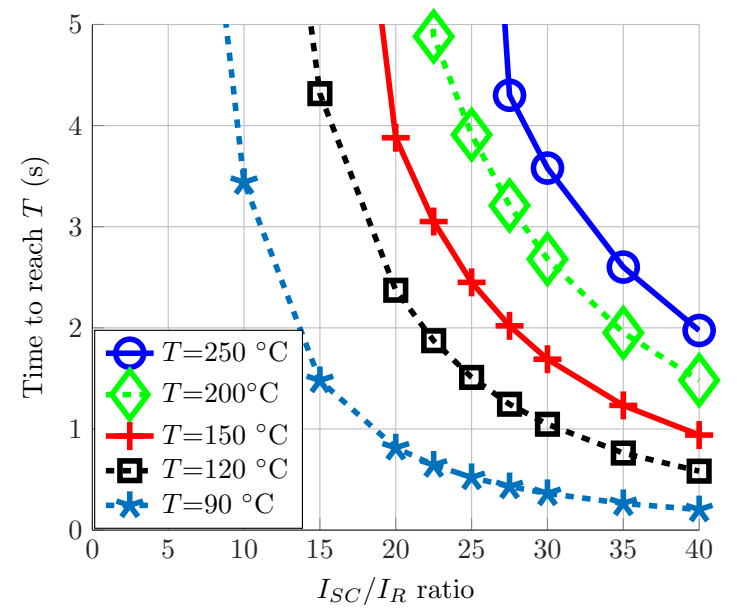

Figure 8: Core temperature profiles for different three-phase short-circuit levels.

During this time, the cable cores and screens temperatures must remain below a maximum allowed limit to prevent any excessive damage of the dielectric insulation. The results of this study are shown in Fig. 8 It may be observed that the maximum fault current that the cable is able to withstand for the specified duration of 5 seconds is equal to 25 times the rated current $I_{R}$. When the fault current is equal to 30 times the rated current, the cable temperature increases indeed to over $250{ }^{\circ} \mathrm{C}$ in about 3.5 seconds, thus failing to meet the requirements established in IEC standard 60949. However, it may be important to mention as well that not meeting this requirement does not lead automatically to an excessive cable damage, as it is expected that protection schemes would have triggered the breakers in a shorter amount of time. For instance, in the case where the short-circuit fault current is sufficiently high, the primary protection system can usually clear the corresponding fault in approximately $200 \mathrm{~ms}$. Longer fault duration may be permitted if they are handled by the back-up protection system. According to 29, fault durations range often between $0.5 \mathrm{~s}$ and $1 \mathrm{~s}$, which is significantly shorter than the duration of $5 \mathrm{~s}$ recommended in IEC standard 60949 and which, therefore, may be considered as quite conservative. 


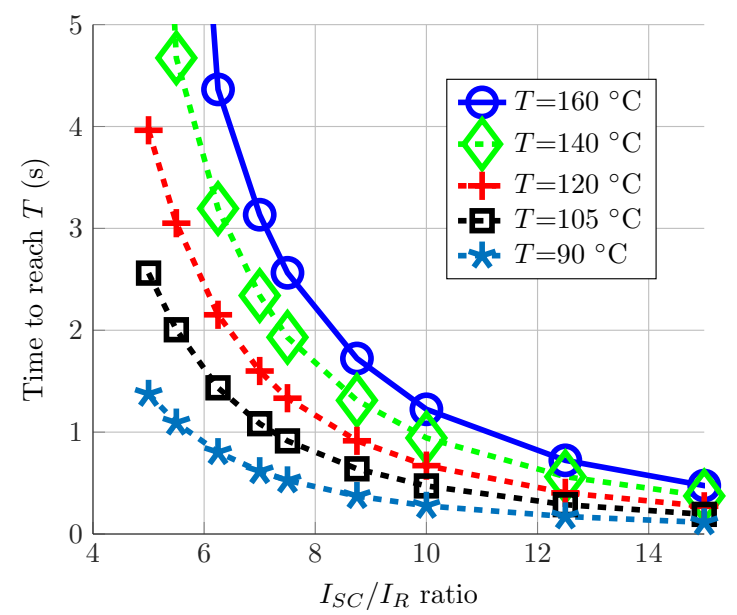

Figure 9: Screen temperature profiles for different single phase short-circuit levels.

370 short-circuit are shown in Fig. 9. They represent the copper screen temperatures, as opposed to the core temperatures which were presented in the previous section. The curves are similar to these presented in Fig. 8, which was expected since adiabatic conditions can almost be considered. It may be observed that, ditions considered in this study. This stems from a lower maximum allowed limit of $160{ }^{\circ} \mathrm{C}$ due to the neighboring sheath material (PE), and to the smaller cross-section of the copper screen. Under these conditions, the cable is only able to withstand single-phase fault currents of up to 6 times the rated current for the specified duration of $5 \mathrm{~s}$. This may impose single-phase short-circuits as the critical design conditions if this scenario is to be included in the cable sizing requirements which, however, do not seem to be usually the case.

\subsubsection{Summary}

As described in Sections 5.1.1 and 5.1.2, a single-phase short-circuit between 385 a conductor and its copper screen would represent the critical design conditions for the cable sizing. However, this scenario does not seem to be usually taken 
into account in cable sizing requirements. If it is not, the results of the threephase short-circuit scenario showed that the cable could withstand up to 25 times its rated current $I_{R}$. In terms of short-circuit level at $20 \mathrm{kV}$, this represents approximately 250 MVA and corresponds to a medium strength grid [28]. Hence, the three-phase short-circuit may constitute the critical design conditions in terms of cable sizing for regions where the grid is relatively strong (e.g. in the WaveHub (UK) [30 and bimep (Spain) 31] test sites areas), whereas for weaker grids (e.g. in the AMETS test site area (Ireland) 32]), the cable sizing may be limited by the normal conditions. To conclude this section, it may, however, be important to mention as well that two other criteria, which were out of the scope of the presented study, namely the voltage drop along the cable and the Joule losses within the cable, must also be considered in the cable sizing requirements and may also constitute the critical design conditions. Hence, further work should be performed on the compliance to these criteria.

\subsection{Numerical modeling fineness analysis}

As described in the introduction, one of the objective of this paper is to present a quantitative assessment of the error level between the three different numerical models presented here. This comparative analysis is detailed in the second part of this section while the first part describes the comparative analysis between the non-linear 2D FEM model and the experimental results.

\subsubsection{Comparison of the non-linear 2D FEM model with the experimental re- sults}

As mentioned previously, 3D FEM models are computationally-intensive, and especially those combining different physics, as it is the case here. Hence, they are reduced to $2 \mathrm{D}$ models whenever possible. In this section, the results of a comparative analysis between the experiments and the 2D FEM model including non-linear thermal characteristics are detailed. This analysis was intended to validate the relevance of using a $2 \mathrm{D}$ model instead of a more computationally intensive, 3D one. It may be recalled that the mixing ratio between the filler 
in the air being unknown, simulations had to be performed for several values of this ratio.

Case 1: steady-state DC current. The temperature results are shown in Table 2 (for the core temperature) and in Table 3 (minimum and maximum temperatures for the external sheath). As expected, a filler solely made of PE seems to slightly underestimate the conductor core temperature while a filler solely composed of air shows an overestimated temperature. Even if this has to be refined with additional experiments, the temperature simulated with the mixed filler matched with the expected results, i.e. the core temperature is only slightly higher than the measurements. Based on this observation, a mixed filler was retained in the 2D FEM models (linear and non-linear), with thermal conductivity $\kappa_{f}(T)=0.5 \times \kappa_{P E}(T)$ and thermal capacity $C_{f}(T)=$ $0.5 \times C_{p P E}(T)$. The simulation results show nevertheless good agreement with the experiments. The slight observed error (less than $2{ }^{\circ} \mathrm{C}$ ) is assumed due to be mainly due to the fact that the edge effects were neglected.

Table 2: Core temperature of the powered phase for Case 1

\begin{tabular}{ll}
\hline Value $\left({ }^{\circ} \mathrm{C}\right)$ & Description \\
\hline $54.1^{*}$ & Temp. derived from the 4-point probes method \\
\hline $53.5^{*}$ & Temp. with full PE filler \\
\hline $61.5^{\#}$ & Temp. with full air filler \\
\hline $54.8^{\#}$ & Temp. with mixed filler \\
\hline * Experimental measurements. \\
\# FEM simulation results.
\end{tabular}

The temperature distribution around the cable external sheath, where the thermocouples are positioned, as obtained from both the experimental data and from the numerical simulations, is presented in Fig. 10. Once again, good agreement is observed between the experimental and the simulated results. 


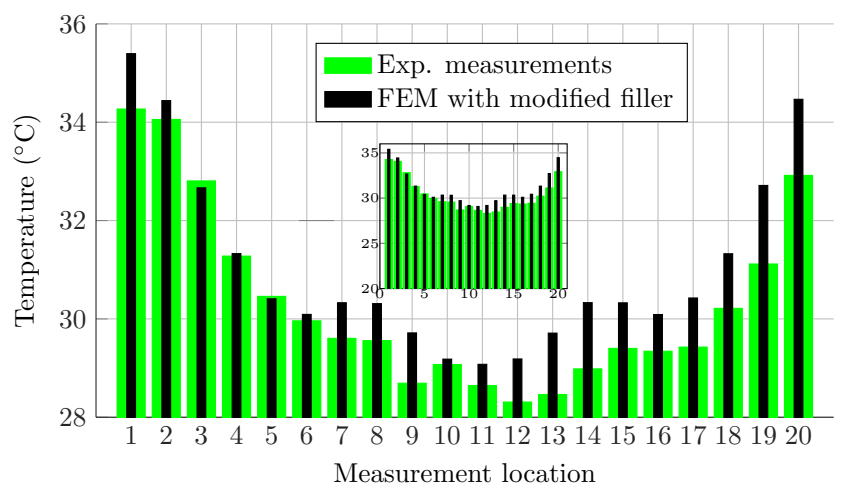

Figure 10: External sheath temperature: experimental and numerical results

Table 3: Maximum and minimum temperatures of the external sheath for Case 1

\begin{tabular}{cl}
\hline Value MIN / MAX $\left({ }^{\circ} \mathrm{C}\right)$ & Description \\
\hline $28 / 34^{*}$ & Temp from TCs measurements \\
\hline $29 / 35^{*}$ & Temp. with full PE filler \\
\hline $29 / 36^{*}$ & Temp. with full air filler \\
\hline $29 / 35.5^{*}$ & Temp. with mixed filler \\
\hline \hline
\end{tabular}

\footnotetext{
* Experimental measurements.

\# FEM simulation results.
}

Case 2: pulsed DC current. The core temperature results for Case 2 are presented in Fig. 11 and Fig. 12. They show the results obtained with the non-linear 2D FEM model (referred to as "FEM" in the figure) and those obtained by measuring the voltage and current in the powered phase(s) (referred to as "T from V, I measurements"). One can remark the good agreement between the experiments and the simulated temperatures, even during the most important variations of the current profiles. Such results show that the model simulated accurately the losses during the heating process and that the nonlinear properties of the cable materials are properly determined, both for the 


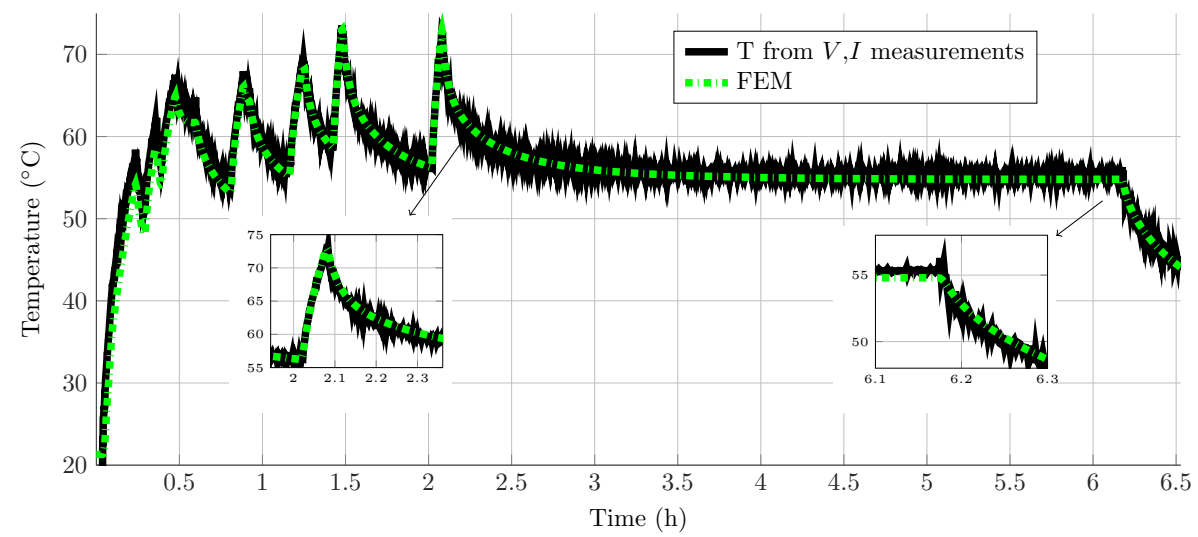

Figure 11: Measured and simulated temperature profiles (Case 2, 1 powered phase)

electrical and thermal problems. Regarding passive cooling, the FEM model is able to correctly reproduce the temperature decrease, which suggests that the heat transfer from the copper core to the external sheath is also well simulated. Such observations, in addition to those already discussed, reinforce the validity of the non-linear 2D FEM model. This observation applies to the entire range of typical operating temperatures that should remain below $90^{\circ} \mathrm{C}$ under normal conditions (as opposed to short-circuit conditions). One can remark the slight differences which arise when the temperature reaches $80{ }^{\circ} \mathrm{C}$. These can be explained by the physical properties of the XLPE insulation material that shows strong non-linearities of the thermal resistivity and of the heat capacity between $80{ }^{\circ} \mathrm{C}$ and $120{ }^{\circ} \mathrm{C}[33$. However, the temperature difference does not exceed $2.5{ }^{\circ} \mathrm{C}$. Hence, it was not deemed necessary to adjust or to refine the thermal properties of XLPE using, for example, a differential scanning calorimeter (DSC) and a guarded hot plate (GHP) method.

Concerning the external sheath temperature presented in Fig. 13 , it can be observed that the model seems to overestimate the temperature (up to $2{ }^{\circ} \mathrm{C}$ ). However, the overall temperature trend over time is very similar between the experiments and the simulations. Hence, these results suggest that the simulations do not fully reflect the measurements in terms of magnitude. As already 


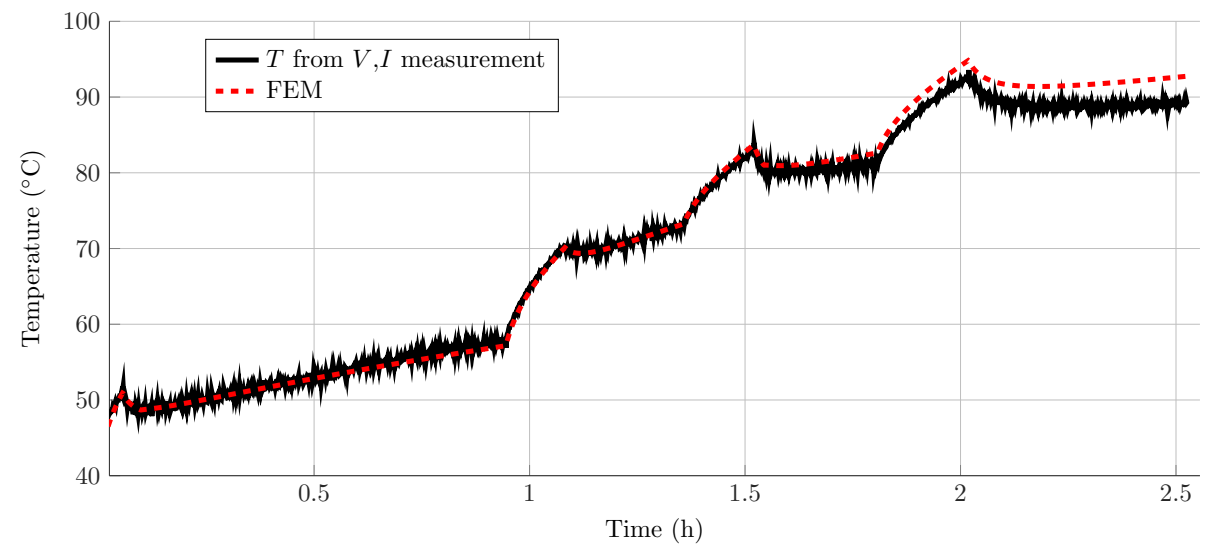

Figure 12: Measured and simulated temperature profiles (Case 2, 3 powered phases)

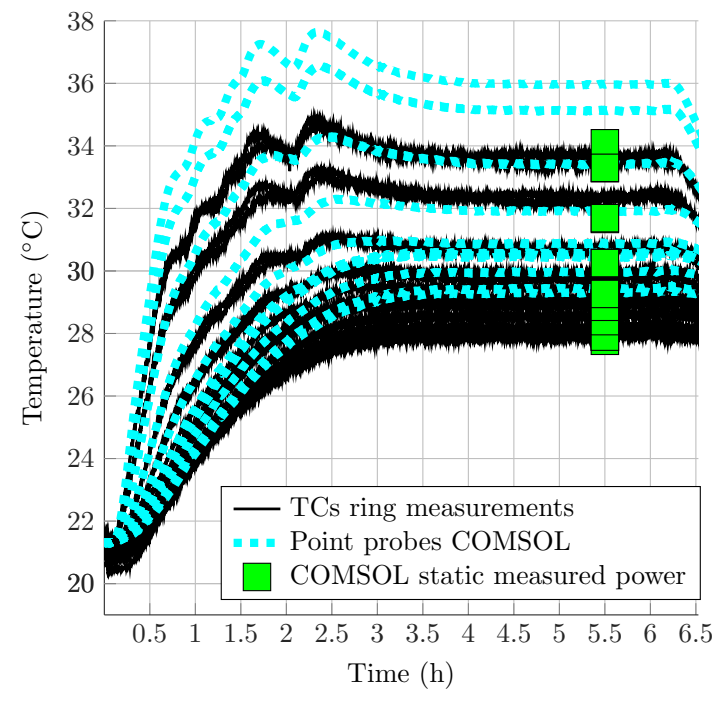

Figure 13: External sheath temperature. 1) Black profiles show the temperature measurements with TCs, 2) Dotted cyan profiles represent the results obtained with the non-linear 2D FEM model and 3) Green squares show the temperature for a pure thermal FEM simulation in static conditions using measured power as a source term.

mentioned earlier, this error may stem from the fact the edge effects at each extremity of the cable were assumed negligible, thus resulting in an overestimation 
Case 3: steady-state AC current. This third study focuses on the characterization of the cable under AC steady-state conditions. The main objective of this test is to verify that the model accurately predicts the losses that, once again, are needed to compute the correct heat flux in the cable and its temperature evolution. This approach allows to evaluate the losses simulations due to the skin effect, as well as the screen and armour losses. In this case study, all the three phases were powered. Due to the rating limitations of the experimental current source, the injected current was approximately equal to $200 \mathrm{~A}_{R M S}$ per phase. Such current level is obviously lower than the rated current $I_{R}$ but this does not prevent from performing a comparative study between the FEM simulations and the experimental measurements. Table 4 shows the results obtained with the non-linear 2D FEM model and the experimental measurements, as well as some available manufacturer data (referred to as MD). It can be observed that the differences between all these data types are reasonable.

sommary. Despite some inaccuracies that have been observed between the non-linear 2D FEM model and the experimental measurements, the results indicate that considering a $2 \mathrm{D}$ model is sufficiently precise for performing the electro-thermal analyses described earlier in this paper. For example, it was observed that the 2D FEM model provided good results for the core temperatures as well as for the external sheath temperature. It is worth mentioning that the differences between our simulations and the measurements on our short cable are assumed to be due to the fact that edge effects were neglected in the $2 \mathrm{D}$ FEM model. Note that such differences would have been drastically reduced considering a longer cable, which would be the case in a typical WEC farm installation, where cable lengths of few hundreds of meters to few tens of kilometers are used. Based on this assumption, we can neglect edge effects and axial heat flows and assume that the non-linear 2D FEM model is sufficiently accurate to estimate the cable electro-thermal behavior that could be installed in a WEC farm. Based on these observations, the model presented in this pa- 
Table 4: Experimental measurements, FEM simulation results and manufacturer data (MD) (Case 3)

\begin{tabular}{lcccl}
\hline Parameters & FEM & Exp. & MD & Description \\
\hline$P(\mathrm{~W} / \mathrm{m})$ & 25.04 & 24.68 & - & Total power losses ${ }^{a}$ \\
\hline$R(\mu \Omega / \mathrm{m})$ & 195 & $201^{b}$ & 196.4 & Resistance of a phase ${ }^{c}$ \\
\hline$L(\mathrm{nH} / \mathrm{m})$ & 445 & $411^{*}$ & 400 & Inductance $^{\circ}$ \\
\hline$P_{\text {cores }}(\mathrm{W} / \mathrm{m})$ & 24.55 & - & - & Losses in copper cores \\
\hline$P_{\text {armour }}(\mathrm{W} / \mathrm{m})$ & 0.07 & - & - & Losses in the armour \\
\hline$P_{\text {screens }}(\mathrm{W} / \mathrm{m})$ & 0.46 & - & - & Losses in copper screens \\
\hline \hline
\end{tabular}

* Experimental measurements in steady-state. Mean value.

${ }^{a}$ The total power was divided by the cable length.

$b$ Total equivalent resistance of one phase of the cable, including skin effects in the conductor cores, screens losses and armour losses.

${ }^{c}$ Resistance values given for a temperature of $20{ }^{\circ} \mathrm{C}$

per is deemed valid, and it was considered as a benchmark for the two other numerical models, i.e. the IEC standard-based model and the linear 2D FEM model. It is however important to recall that the validity domain of the model, in terms of temperature, does not exceed $90^{\circ} \mathrm{C}$ : this model cannot thus be qualified as experimentally validated for short-circuit studies. Further experimental work focusing on high temperatures should therefore be carried out, which was however impossible to do as part of this study, due to the experimental means limitations.

\subsubsection{Comparison between the three cable numerical models}

In Fig. 7, the IEC standard-based model is referred to as "IEC method", the linear 2D FEM model as "FEM + IEC data", as it may be recalled that it includes the linear thermal characteristics provided in IEC standards 60287-1-1 and 60287-2-1, and the non-linear 2D FEM model as "FEM + non-linear data". 
Regarding the difference between the IEC standard-based model and the non-linear 2D FEM model, it is important to mention that the formulae pro-

in the zone where the cable thermal characteristics are highly non-linear, e.g between $80{ }^{\circ} \mathrm{C}$ and $120{ }^{\circ} \mathrm{C}$ in our case, which automatically reduces the heat transfer from the core to the external sheath. Otherwise, if the target operating 
temperature is outside the highly non-linear zone, the discrepancy between the

linear thermal characteristics may be used. It is also interesting to note that the simulated temperatures may be either under- or overestimated by the linear 2D FEM model, as shown in Fig. 7b and Fig. 7f. respectively. In addition, compared to the IEC standard-based model, the 2D FEM models presented in considering a 20 WECs farm, the maximum temperatures are equal to $76{ }^{\circ} \mathrm{C}$ when the IEC standard-based model is used, $84{ }^{\circ} \mathrm{C}$ when the linear 2D FEM model is used and $90{ }^{\circ} \mathrm{C}$ when the non-linear 2D FEM model is considered. This phenomenon may stem from the measure considered here (i.e. the RMS value of the RMS current profile), but also from the fact that, as already mentioned earlier, IEC standard 60287-1-1 generally underestimates temperature (very) significantly. In any case, adopting an approach which underestimates the core temperature is of course to be avoided to prevent any risks of overheating. It is therefore important to either develop an enhanced IEC model, or to use a intensive which may raise computing time issues if an optimization algorithm requiring a large number of iterations using the electro-thermal model is selected. Hence, further work will be undertaken regarding the level of modeling fineness required for such optimization studies, and especially on the development of a suitable electro-thermal cable model for computationally-intensive optimization studies.

\section{Conclusion}

This paper described preliminary cable sizing studies focusing on the electrothermal response of a submarine cable to be used as an export cable in a wave energy farm. It was shown that a current profile whose RMS value is $9 \%$ greater than the rated current $I_{R}$, and whose maximum value is equal to approximately 2.5 times this rated current, may be safely injected in the cable without violating 
the thermal constraints. Conversely, this implies that a wave farm export cable can be safely sized to a dramatically lower value than the maximum current of the farm, thus leading to potentially significant savings. Hence, considering more realistic, dynamic conditions at the wave farm planning stage is recommended. It was also shown that short-circuit conditions may become the critical design conditions for areas with a short-circuit level of at least 250 MVA which, however, may not be the case for a large share of wave energy abundant regions such as the west of Ireland. As for the latter, normal conditions (i.e. without short-circuit) constitute the critical design conditions. The paper also focused on the level of numerical modeling fineness required for cable sizing studies. A comparative analysis was carried out between three cable numerical models proposed in this study, the most refined of which was validated against experimental data. It was concluded that the proposed IEC standard-based model led to an important temperature underestimation ranging between $10 \%$ and $17 \%$ of the maximum allowed temperature $\left(90^{\circ} \mathrm{C}\right)$ and needed to be enhanced to be fully relevant in wave energy applications. Hence, using a FEM-based model, including potentially non-linear thermal characteristics (if the targeted temperature is within the highly non-linear zone of these characteristics) seems to remain the safest option. However, the computational burden linked to $\mathrm{u}-$ sing FEM-based models may represent an issue for certain optimization studies where a large number of iterations is required. Investigating further this issue, developing a suitable electro-thermal cable model, and testing its performances, both in terms of error level and computational burden, will be the focus of future work. Following this, detailed techno-economic optimization studies on the sizing of a wave farm export cable will be carried out.

\section{Acknowledgment}

The research work presented in this paper was conducted in the frame of the EMODI project (ANR-14-CE05-0032) funded by the French National Agency of Research (ANR), and continued in the frame of the BlueGrid project, funded 
by the Brittany Regional Council. Complementary funding from ENS-Rennes was also provided. All these organizations are greatfully acknowleged. It is worth mentioning that this work would not have been possible without the help of École Centrale de Nantes that manages the SEM-REV test site and provided a sample of their export cable. The authors would also like to thank Thomas

Soulard (LHEEA lab., École Centrale de Nantes), Didier Trichet, Guillaume Wasselynck, Jean-Christophe Olivier and Franck Judic (all with the IREENA lab., Université de Nantes) for valuable discussions and occasional assistance.

\section{References}

[1] C. Kost, S. Shammugam, V. Juelch, H.-T. Nguyen, T. Schlegl, Levelized cost of electricity renewable energy techologies, Tech. rep., Fraunhofer ISE (2018).

[2] International levelised cost of energy for ocean energy technologies, Tech. rep., IEA Technology Collaboration Programme for Ocean Energy Systems (OES) (2015).

[3] Wind turbine technology and operations factbook, Tech. rep., E.ON Climate \& Renewables (2013).

[4] S. Gasnier, V. Debusschere, S. Poullain, B. François, Technical and economic assessment tool for offshore wind generation connection scheme: Application to comparing $33 \mathrm{kV}$ and $66 \mathrm{kV}$ ac collector grids, in: $18^{\text {th }} \mathrm{Eu}-$ ropean Conference on Power Electronics and Applications (EPE'16 ECCE Europe), 2016, pp. 1-8. doi:10.1109/EPE.2016.7695269.

[5] A. MacAskill, P. Mitchell, Offshore wind - an overview, Wiley Inter620 disciplinary Reviews: Energy and Environment 2 (4) (2013) 374-383. doi:10.1002/wene.30

[6] F. Sharkey, E. Bannon, M. Conlon, K. Gaughan, Maximising value of electrical networks for wave energy converter arrays, International Journal of Marine Energy 1 (2013) 55-69. doi:10.1016/j.ijome.2013.06.002. 
[7] D. Bull, M. Baca, B. Schenkman, Electrical cable utilization for wave energy converters, Journal of Ocean Engineering and Marine Energy 4 (2) (2018) 171-186. doi:10.1007/s40722-017-0102-x.

[8] A. Blavette, D. O'Sullivan, T. Lewis, M. Egan, Dimensioning the equipment of a wave farm: Energy storage and cables, in: IEEE Int. Conf. on Ecological Vehicles and Renewable Energies (EVER), 2013.

[9] A. Matine, C.-H. Bonnard, A. Blavette, S. Bourguet, F. Rongère, T. Kovaltchouk, E. Schaeffer, Optimal sizing of submarine cables from an electrothermal perspective, in: $12^{\text {th }}$ European Wave and Tidal Energy Conference (EWTEC), 2017, pp. 1-7.

[10] IEC standard 60287-1-1, Electric cables Calculation of the current rating -

1. Part 1-1: Current rating equations (100\% load factor) and calculation of losses - General (2006).

URL https://webstore.iec.ch/publication/1265

[11] IEC standard 60853-1, Calculation of the cyclic and emergency current

$640 \quad$ rating of cables. Part 1: Cyclic rating factor for cables up to and including 18/30(36) kV (1985).

URL https://webstore.iec.ch/publication/4016

[12] École Centrale de Nantes, The SEM-REV website (2018).

URL https://sem-rev.ec-nantes.fr/english-version/

[13] IEC standard 60949, Calculation of thermally permissible short-circuit currents, taking into account non-adiabatic heating effects (1988).

URL https://webstore.iec.ch/publication/4016

[14] IEC standard 60287-2-1, Electric cables Calculation of the current rating - Part 2-1: Thermal resistance - Calculation of thermal resistance (2006). URL https://webstore.iec.ch/publication/1274

[15] J. Wen, Heat capacities of polymers, in: Physical properties of polymers handbook, Springer, 2007, pp. 145-154. 
[16] COMSOL INC., The COMSOL Multiphysics@) website (2018).

URL https://www.comsol.fr/products

655

[23] IEC standard 60502, Power cables with extruded insulation and their ac-

प cessories for rated voltages from $1 \mathrm{kV}(\mathrm{Um}=1,2 \mathrm{kV})$ up to $30 \mathrm{kV}(\mathrm{Um}=$ $36 \mathrm{kV})(1988)$.

URL https://webstore.iec.ch/publication/2274 
[24] M. Pays, Câbles de transport d'énergie - Technologies, Caractéristiques, (In French), Techniques de l'ingénieur Énergies - Réseaux électriques et applications (D4520) (1994) 1-32.

[25] O. Anaya-Lara, J. O. Tande, K. Uhlen, K. Merz, Offshore Wind Energy

a Technology, John Wiley \& Sons, Ltd, Chichester, UK, 2018. doi:10.1002/

685

[26] R. Walling, E. Gursoy, B. English, Current contributions from type 3 and type 4 wind turbine generators during faults, in: IEEE PES Transmission and Distribution Conference and Exposition (T\&D), 2012, pp. 1-6.

[27] J. D. Glover, M. S. Sarma, T. Overbye, Power System Analysis and Design, Cengage Learning, 2011, ISBN : 978-1-111-42577-7.

[28] A. Blavette, Grid integration of wave energy \& generic modelling of ocean devices for power system studies, Ph.D. thesis, University College Cork (2013).

[29] E. Dorison, Modélisation et dimensionnement des liaisons souterraines.

[30] WaveHub test site.

URL https://www . wavehub.co.uk/

[31] Bimep test site.

URL https://bimep.com/

[32] AMETS test site.

URL http://www . oceanenergyireland.ie/TestFacility/AMETS

[33] X. Qi, S. Boggs, Thermal and mechanical properties of EPR and XLPE cable compounds, IEEE Electrical Insulation Magazine 22 (3) (2006) 19-24. doi:10.1109/MEI.2006.1639026.

705

[34] A. Sedaghat, F. de Len, Thermal analysis of power cables in free air: Evaluation and improvement of the IEC standard ampacity calculations, 
IEEE Transactions on Power Delivery 29 (5) (2014) 2306-2314. doi:

10.1109/TPWRD. 2013.2296912. 